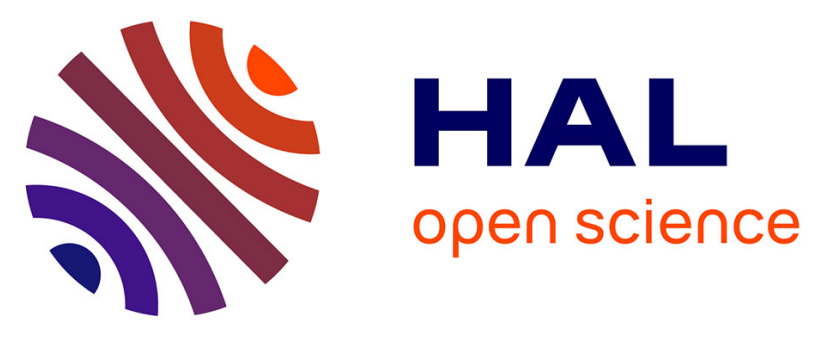

\title{
Numerical Study of the Biomechanical Behaviour of the Different Implantation Methods of the Reverse Shoulder Replacement
}

\author{
Salah Mebarki, Benaoumeur Aour, Etienne Malachanne, Franck Jourdan, \\ Abdel Hakem Belaghit, Abdelkader Gasmi
}

\section{- To cite this version:}

Salah Mebarki, Benaoumeur Aour, Etienne Malachanne, Franck Jourdan, Abdel Hakem Belaghit, et al. Numerical Study of the Biomechanical Behaviour of the Different Implantation Methods of the Reverse Shoulder Replacement. Journal of Biomimetics, Biomaterials and Biomedical Engineering, 2019, 43, pp.54-66. 10.4028/www.scientific.net/JBBBE.43.54 . hal-02419732

\author{
HAL Id: hal-02419732 \\ https://hal.science/hal-02419732
}

Submitted on 19 Dec 2019

HAL is a multi-disciplinary open access archive for the deposit and dissemination of scientific research documents, whether they are published or not. The documents may come from teaching and research institutions in France or abroad, or from public or private research centers.
L'archive ouverte pluridisciplinaire HAL, est destinée au dépôt et à la diffusion de documents scientifiques de niveau recherche, publiés ou non, émanant des établissements d'enseignement et de recherche français ou étrangers, des laboratoires publics ou privés. 


\title{
Numerical Study of the Biomechanical Behaviour of the Different Implantation Methods of the Reverse Shoulder Replacement
}

\author{
Salah Mebarki ${ }^{1, a^{*}}$, Benaoumeur Aour ${ }^{1, b}$, Etienne Malachanne ${ }^{2, c}$, \\ Franck Jourdan ${ }^{3, \mathrm{~d}}$, Abdel Hakem Belaghit ${ }^{1, \mathrm{f}}$, Abdelkader Gasmi ${ }^{\mathrm{c}, 4}$ \\ ${ }^{1}$ Laboratory of Applied Biomechanics and Biomaterials, Department of Mechanical Engineering, \\ National Polytechnic School of Oran - Maurice Audin, Oran, 3100, Algeria \\ ${ }^{2}$ Laboratoire de Mécanique et Génie Civil (LMGC), Université de Montpellier, CNRS, 34090 \\ Montpellier, France \\ ${ }^{3}$ C2MA, IMT Mines Ales, Université de Montpellier, 30100 Ales, France \\ ${ }^{4}$ Clinique Caducée Bir El Djir, 31000, Oran, Algérie \\ a*mebarkiu_salah@yahoo.fr , bbenauomeur.aour@enp-oran.dz
}

Keywords: Biomechanics, Shoulder prosthesis; Finite elements; Abduction; Stresses; BIO-RSA.

\begin{abstract}
Despite the widespread use of reverse total shoulder arthroplasty, there is still a problem of conflict between the polyethylene cup of the prosthesis and the scapula, which over time causes the phenomenon of notching. In order to circumvent this problem correctly, several innovations have been proposed regard to the implementation method. In this context, the aim of this work is to study the biomechanical behavior of new implantation methods using different glenoid configurations in order to avoid the notching phenomenon between the cup and the scapula. The study was carried out using virtual prototypes of the shoulder prosthesis assembly. Forum CT scan images, threedimensional models of shoulder bones were reconstructed. The implantation of the prosthesis in 3D model was performed in collaboration with an experienced surgeon from the Caduceus Clinic (Oran, Algeria). The obtained models were imported to finite element calculation software. After the validation of the numerical model using the literature results, we assessed the biomechanical behavior of four implantation methods under the same boundary conditions and abduction movements. Among the used methods, the BIO-SR lateralization method offers significant biomechanical advantages in terms of the forces applied to the glenoid during the abduction movement.
\end{abstract}

\section{Introduction}

The reverse shoulder arthroplasty (RSA) was initially developed by Paul Grammont to address the problem of omarthrosis associated with rotator cuff failure for people over 70 years old [1]. This arthroplasty has been used as a surgical treatment with positive clinical results to relieve pain and restore shoulder function for patients with severe rotator cuff arthropathy and glenohumeral arthritis $[2,3]$. These indications have expanded considerably and now include many more complex etiologies such as rheumatoid arthritis, complex fracture fixation, scapular belt tumors and revision of failed anatomical arthroplasty [4]. Clinical successes of the restoration abduction function have given confidence to extend the indications of the reverse shoulder prosthesis to proximal humeral fractures, deficient bone with intact cuff, cancer and many others $[5,6]$. Since 2007, shoulder arthroplasty has been developed at the same rate as knee and hip arthroplasty or even faster [7], and the RSA has recently been estimated at $42 \%$ of all shoulder replacements [8].

Paul Grammont revolutionized the RSA with the introduction of a new implant design. The Grammont system is based on four principles that he identified to ensure stabilization while allowing the deltoid to compensate for the deficient or absent rotator cuff $[9,10,11]$. These early designs improved the range of motion in patients with rotator cuff deficiencies by tightening and repositioning the deltoid relative to the fixed center of rotation (COR) of the joint. In addition, the COR medialization increases the lever arm of the deltoid from 20 to 42\% [12]. Although the medialized design certainly improves function in patients with deficient rotator cuff shoulders. The clinical 
results have identified some defects due to a strong medialization of the humerus, including scapula notches, in 40 to $96 \%$ of cases, are observed in the first months [13-15]. These notches are secondary to a mechanical conflict between the humeral cup and the scapula pillar. These complications have as effect a premature deterioration of the (at the cup level) and cause severe and consequent pain for the patient [16]. Polyethylene and metal debris will secondarily cause additional osteolysis [17]. In order to solve this problem, several modifications were made to the design of the Grammont prosthesis. Among which, we find lateralization with the BIO-RSA method, with a lower offset and metal lateralization of the glenosphere [18].

In a previous numerical work [19], the bio-faithful geometry of the shoulder joint and the biocompatibility of the materials used in the lateralized method have been investigated. In the present work, four different implantation methods of reverse shoulder prostheses have been investigated in order to find an adequate solution to the notch problem. The model developed will be validated by comparing the results obtained from the forces observed at the glenoid implant as a function of the humerus abduction angle with those obtained from the models of the literature. The biomechanical behaviors of the four different implantation methods, namely, the normal method (without modification), the BIO-RSA method, that with glenosphere offset of $2 \mathrm{~mm}$ downwards and $3.7 \mathrm{~mm}$ of lateral shift, will be studied. Based on the results obtained, recommendations on the choice of implementation method will be proposed at the end of this work.

\section{3D Reconstruction of the Shoulder Joint}

The model used was generated by processing images from an MRI file, using 3D reconstruction technique through several steps. This method has been detailed in a previous work [19], and will be just recalled in this part. The data conversion method used to develop the finite element model of the shoulder is illustrated in Figure 1. The first step consists in retrieving the data from computerized tomographic sections (CT scan), namely a sequential images file with an interval of $0.5 \mathrm{~mm}$ in "DICOM" format. The radiographic images are transferred to Mimics image processing software. This anatomical representation software allows to visualize the reconstruction of tomographic sections in 3D. It shows a precise model of the shoulder complex made with an accuracy of about one tenth of a millimeter.

The surface generated by Mimics software is not always of good quality. It is common to find anomalies, most often in the form of folds: the surface wraps in place on itself to create a bead. These invisible irregularities disturb the generation of the mesh by creating an infinity of superimposed elements or elements of almost zero size. To correctly circumvent these geometric singularities, we used the RapidForm software to perform a smoothing operation by filling the gaps and rebuilding regular automatic mesh again using NURBS surfaces. The resulting three-dimensional geometric data will be converted into a type data format (Parasolid $x_{-} t$ ) in order to be imported into the ANSYS finite element calculation software.

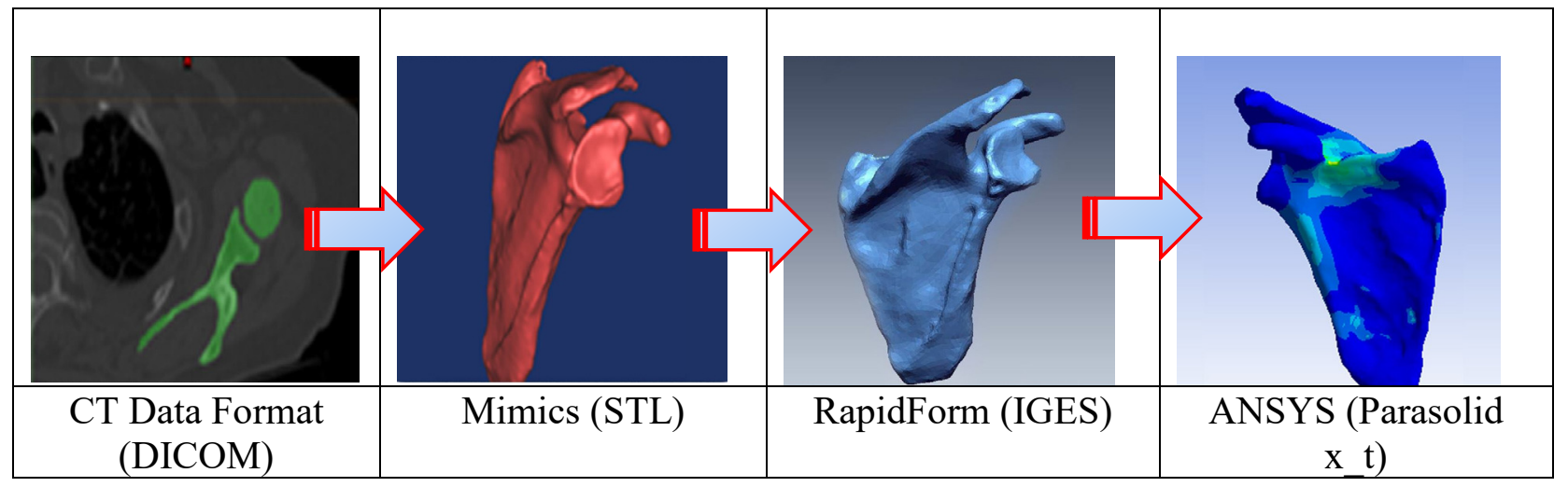

Fig. 1 Illustration of the 3D reconstruction procedure of the bone structure (case of the scapula) 


\section{Finite Element Modeling}

The Delta3 reverse shoulder prosthesis model was chosen for its wide use. The three-dimensional design of this prosthesis was carried out using Solidworks CAD software. The prosthesis was implanted in accordance with the operating protocol which consists of planning the proximal end of the humerus at a cervico-diaphyseal angle of $125^{\circ}$. The glenoid part has been reduced by a vertical plane in order to position the glenoid base. The humeral part of the prosthesis is inserted in press-fit. On the glenoid side, the base plate is fixed with two screws [19]. This prosthesis implantation technique is adopted by experienced surgeons specialized in the placement of this type of prosthesis at the Caduceus Clinic of Oran (Algeria). This implantation will be used in what follow to validate the numerical model (called normal implantation).

\subsection{Boundary conditions}

Large strain kinematics is assumed with transient dynamics conditions. For boundary conditions, the scapula was assumed to be fully constrained, however an abduction movement was imposed on the humerus. With regard to the lifting force of the humerus, as suggested in the literature, it has been assumed that the deltoid is the only muscle used during arm abduction. Then, to estimate the abduction force due to the deltoid muscle, some hypothesis have been done. In particular, the deltoid was considered as a simple one-dimensional viscoelastic element that acts along specific lines. These lines of action are delimited by the insertion of the deltoid muscles which represent the loading points. For some kinds of muscles, such as the deltoid, where there is more than one torque, equivalent insertion points can be defined to determine an "equivalent" acting line. The deltoid was therefore modeled as a set of three active springs and dampers whose ends correspond to muscle anchor points (Figure 2). The muscle contraction is achieved by imposing a preload that has been estimated in order to regain contact forces comparable to those in the literature. The values of damping and stiffness coefficients were chosen to minimize the dynamic effects (see Table 1).

For all the numerical models, a vertical force of $37 \mathrm{~N}$, which represents the weight of the arm, was applied to the gravity center of humerus. Contact conditions with a friction coefficient $\mu=0.07$ were applied between the cup and the glenosphere [20]. All other contacts between the different parts are assumed to be totally linked (bilateral contact).

Table 1 Characteristics of all active springs and damping

\begin{tabular}{c|ccc}
\hline \multirow{2}{*}{ Element } & Damping coefficients & $\begin{array}{c}\text { Stiffness coefficients } \\
(\mathbf{N} / \mathbf{m})\end{array}$ & Preload (N) \\
\hline $\mathbf{1}$ & 500 & 7000 & 314 \\
$\mathbf{2}$ & 500 & 7000 & 317 \\
$\mathbf{3}$ & 500 & 7000 & 312 \\
\hline
\end{tabular}




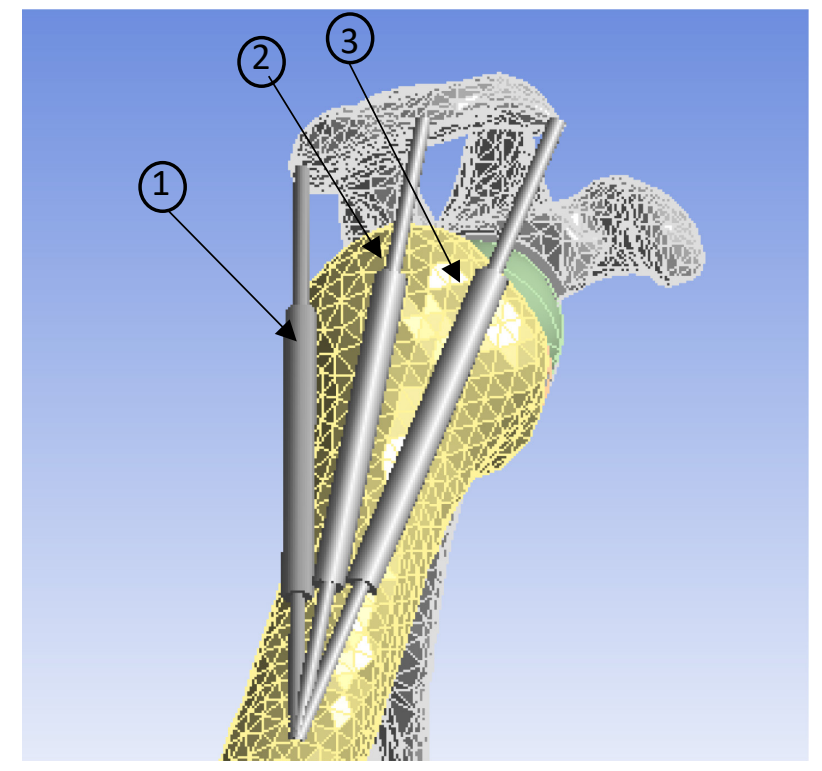

Fig. 2 Illustration of the active spring and damping elements.

\subsection{Material properties}

All solids are considered linear elastic whose material properties are shown in Table 2.

Table 2 Material properties of bons and prothesis élements [21].

\begin{tabular}{|c|c|c|c|c|}
\hline Elements & Materials & $\begin{array}{c}\text { Young's module } \\
\text { E(MPa) }\end{array}$ & $\begin{array}{c}\text { Poisson } \\
\text { coefficient } v\end{array}$ & Density $\left(\mathrm{g} / \mathrm{mm}^{3}\right)$ \\
\hline Humerus & Cortical bone & 8000 & 0.3 & $1.3 \times 10^{-3}$ \\
\hline $\begin{array}{c}\text { Screw and screw } \\
\text { holder }\end{array}$ & Titanium & 110000 & 0.33 & $4.5 \times 10^{-3}$ \\
\hline Glenosphere & Stainless steel & 230000 & 0.3 & $8.01 \times 10^{-3}$ \\
\hline Diaphyseal stem & Titanium & 110000 & 0.33 & $4.5 \times 10^{-3}$ \\
\hline Cup & Polyethylene & 80000 & 0.35 & $9.5 \times 10^{-4}$ \\
\hline
\end{tabular}

\subsection{Space and time discretization}

The discretization of the model was performed using a fine mesh with six-nodes tetrahedral quadratic elements. This mesh is composed of 42994 elements and 75381 nodes (Figure 3). For the time discretization an implicit method of Newmark, with a time step equals to $5 \times 10^{-2} \mathrm{~s}$, has been chosen. The time duration of the calculation has been tuned in order to minimize dynamic effects.

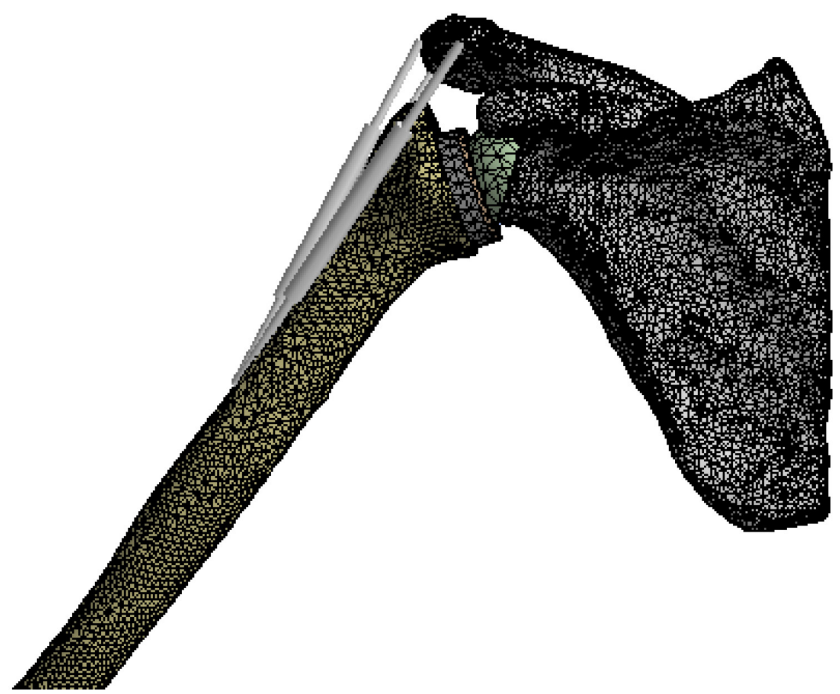

Fig. 3 Finite element mesh used for modeling the behavior of the prosthetic shoulder 


\section{Results and Discussion}

\subsection{Validation of the model}

In order to validate the developed model, we compared the results obtained from the evolution of the forces recorded at the level of the glenoid implant as a function of the humerus abduction angle with those of existing models in the literature (Astier [21], Terrier et al. [22], and Wuelker et al. [23]), as illustrated in Figure 4. It can be seen that the results obtained by the developed model are in good agreement with those of Astier model [21], whereas they overestimate those of Wuelker et al. [23] and underestimate those of Terrier et al. [22]. In addition, almost all models show an increasing slope up to the angle of $90^{\circ}$, with the exception of Astier model, where a slight decrease was observed from the angle of $60^{\circ}$. This difference between the results can be justified by the difference in anatomical models and the types of prostheses used.

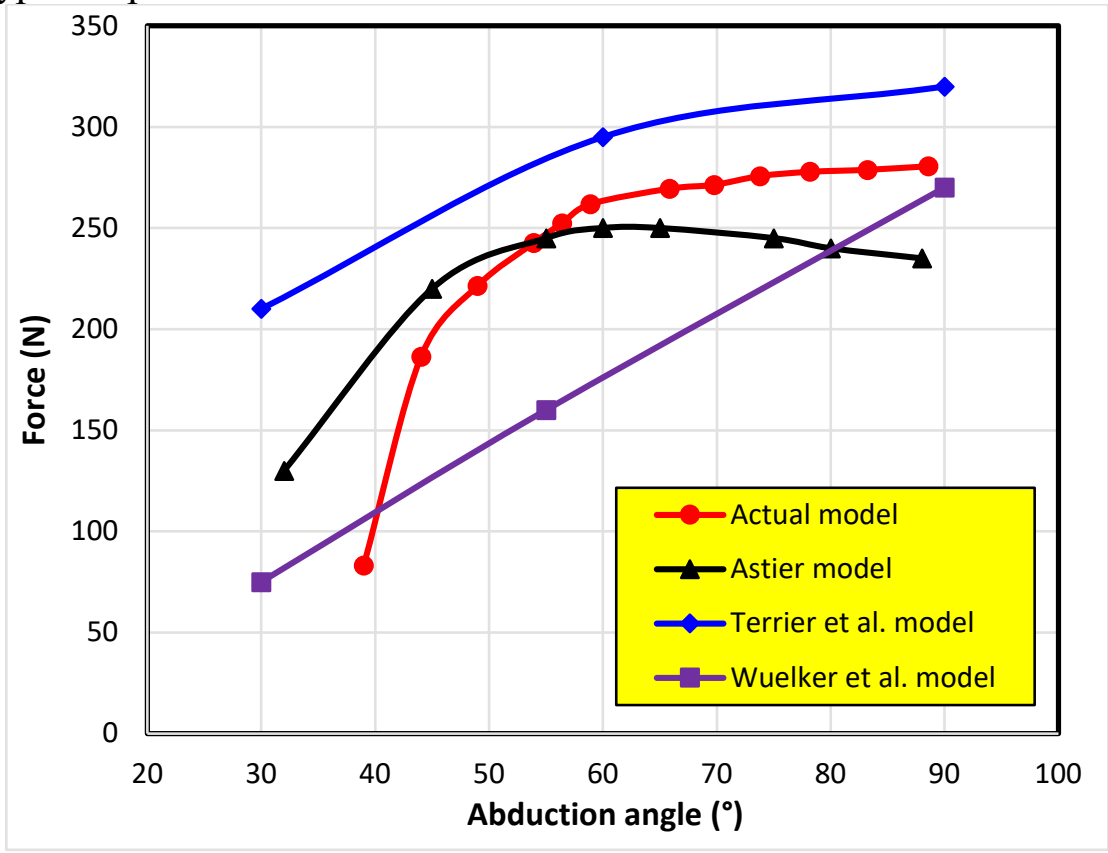

Fig. 4 Evolution of the forces applied on the glenosphere as a function of the abduction angle of the arm

\subsection{Effect of the different implantation methods}

In order to highlight the effects of the implantation methods on the biomechanical behavior of the prosthesis, four different configurations were tested (see Figure 5): (a) a normal implantation with a medialized center of rotation, used to validate the model, (b) implantation with a glenosphere offset from the center of rotation by $2 \mathrm{~mm}$ downwards, (c) implantation with a glenosphere lateralization offset of $10 \mathrm{~mm}$, via bone graft (BIO-RSA) and (d) implantation with a glenosphere lateralization offset of $3.7 \mathrm{~mm}$, via the glenosphere. For the models (a), (b) and (c) glenosphere is considered as a half-sphere. In the fourth configuration (d) the lateral offset is obtained using a sphere with $3,7 \mathrm{~mm}$ de décalage lateral [24]. The models developed are meshed using tetrahedral elements. The same boundary and loading conditions were applied on the four configurations using fine meshes. 


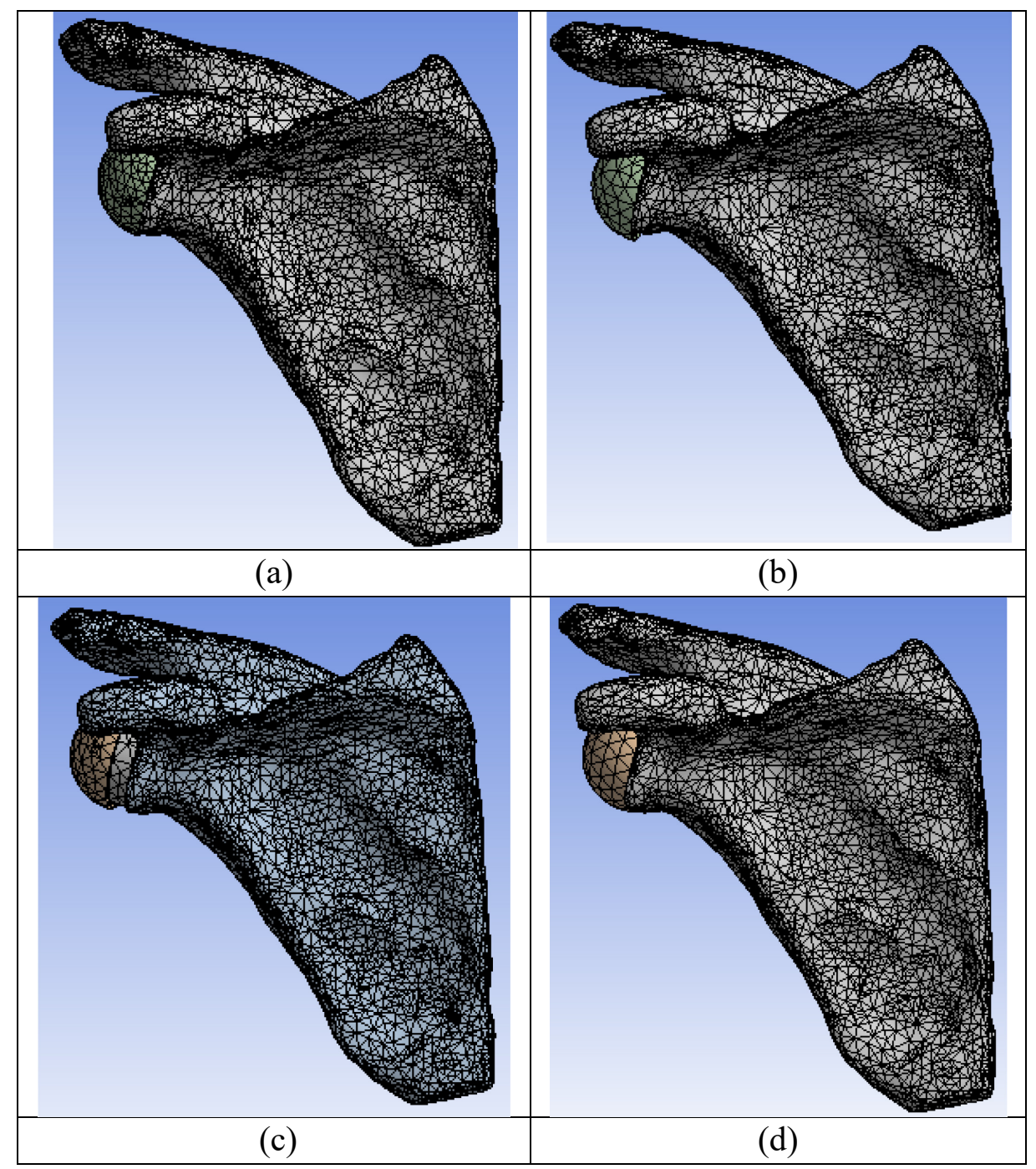

Fig. 5 Illustration of the different configurations: (a) a normal implantation with a medialized center of rotation, (b) implantation with a glenosphere offset from the center of rotation by $2 \mathrm{~mm}$ downwards, (c) implantation with a glenosphere lateralization offset of $10 \mathrm{~mm}$, via bone graft (BIORSA) and (d) implantation with a glenosphere lateralization offset of $3.7 \mathrm{~mm}$, via the glenosphere

\subsubsection{Evolution of the applied forces on the glenosphere}

Figure 6 illustrates a comparison of the evolution of the applied forces on the glenosphere as a function of the abduction angle for the four configurations. It can be seen that the forces are minimal when using a configuration (a) of the normal implantation and they are maximal when the implantation is made with a glenosphere lateralization offset of $3.7 \mathrm{~mm}$ configuration (d). In addition, the behavior closest to that of normal implantation is that of the prosthesis with the BIO-RSA method, i.e., the configuration (c) in Figure 4. 


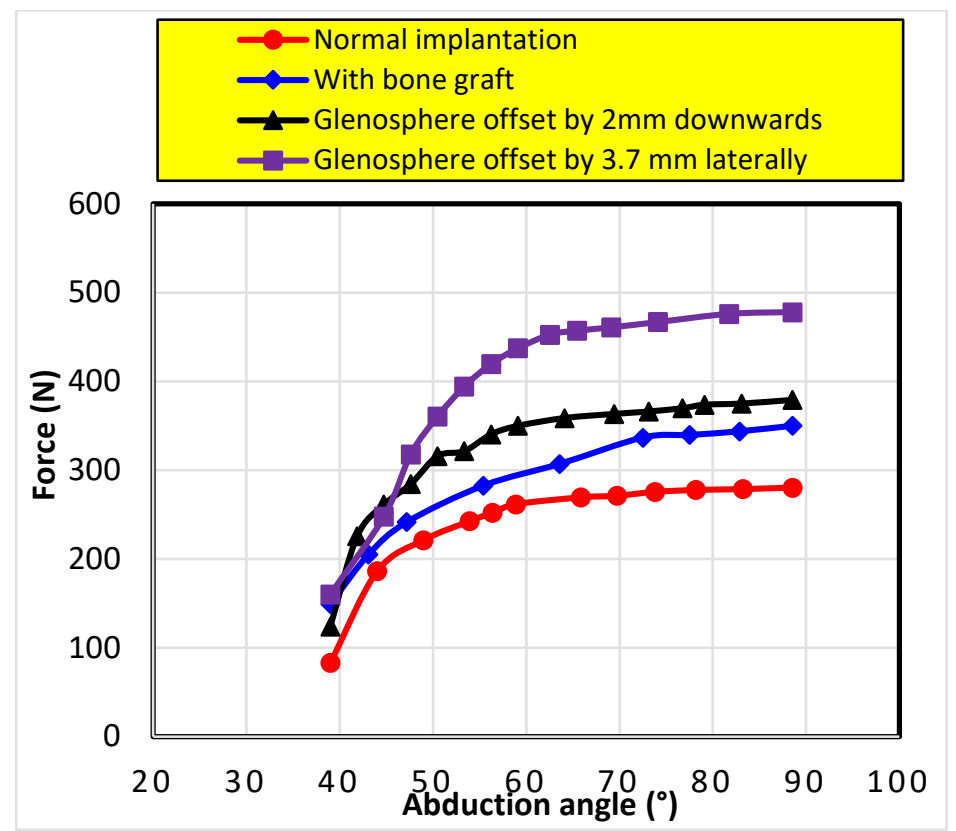

Fig. 6 Evolution of the contact forces applied on the glenoid for the different configurations according to the abduction angle

\subsubsection{Evolution of the von Mises stresses on the contact surface of the glenosphere}

In this section, a comparison of the evolution of the maximum von Mises stresses on the glenosphere contact surface is presented for the four configurations during abduction movement as shown in Figure 7. It can be seen that the values of maximum stresses are obtained in the case of implantation with a normal configuration, whereas the highest values are observed for those of implantation with a glenosphere lateralization offset of $3.7 \mathrm{~mm}$ via the geosphere.

Indeed, this last configuration has two phases: (i) when the abduction angle is less than $62^{\circ}$, the stresses are closed to that of normal implantation; (ii) when the abduction angle is greater than $62^{\circ}$, the maximum stresses obtained are the highest compared to that of the considered configurations.

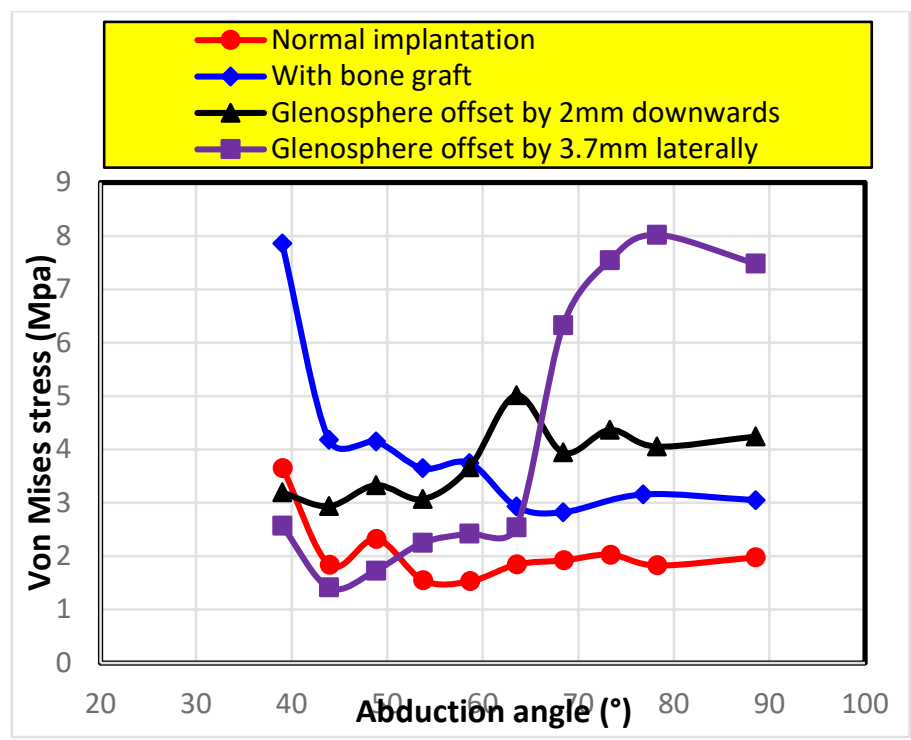

Fig. 7 Evolution of maximum stresses on the glenoid for the different configurations as a function of the abduction angle

Figure 8 shows the distribution of von Mises stresses on the glenosphere at the final position of the abduction motion for the four implantation methods. It is clear that the stresses are higher in the case of the configuration (d), and lower for the normal implantation. Indeed, the behavior closest to that of the normal implantation is that of the configuration with BIO-RSA, method (c). 


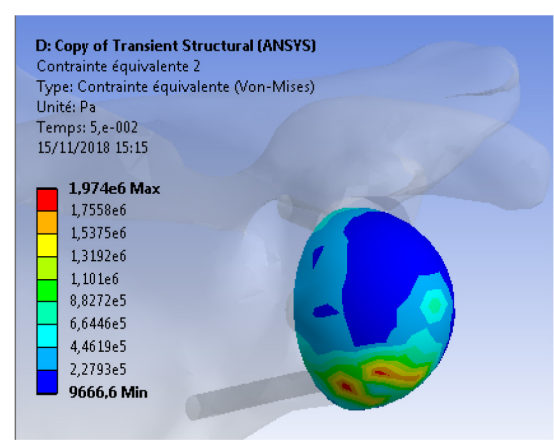

(a)

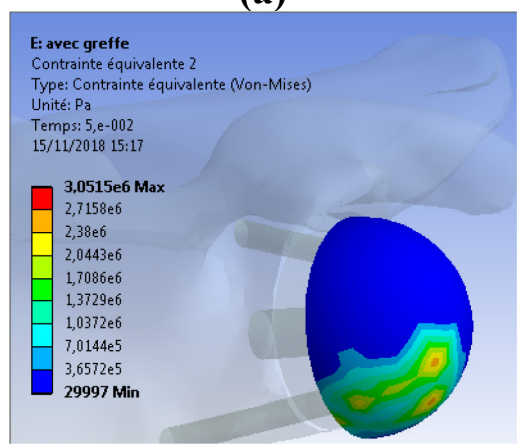

(c)

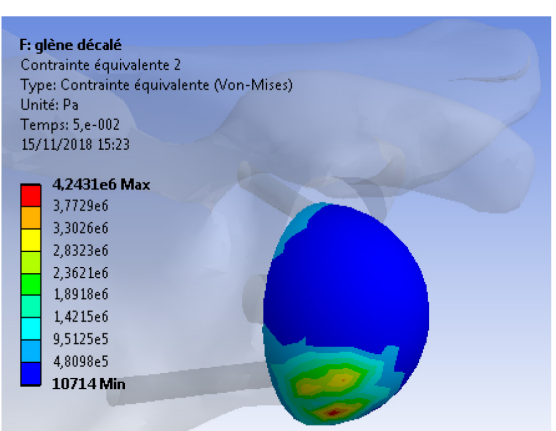

(b)

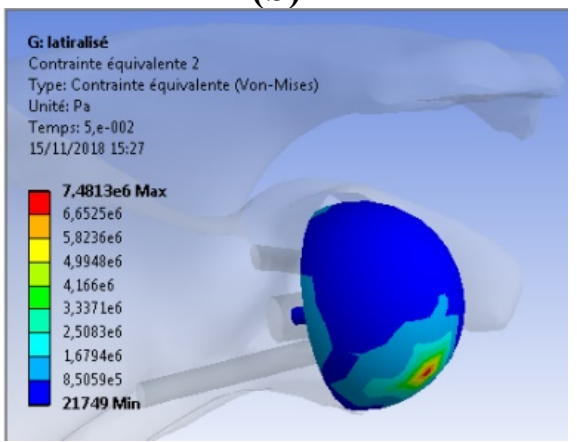

(d)

Fig. 8 Distribution of von Mises stresses on the glenoid for different configurations at the end of the abduction motion

\subsubsection{Evolution of maximum stresses in the support with screws}

In what follows, we present a comparison of the evolution of the maximum von Mises stresses in the support as a function of the abduction angle of the arm as shown in Figure 9. It can be seen that in the initial and final states (at $40^{\circ}$ and $90^{\circ}$ ), the maximum stresses values are obtained in the case of the prosthesis with bone graft (configuration (c)), while the minimum values are obtained by the configuration (d). However, between these two limits (median phase), we found that the minimum stresses values are obtained in the case of normal implantation, whereas the highest stresses values are recorded in the case of configuration (d), when the implantation method with a glenosphere offset from the center of rotation by $2 \mathrm{~mm}$ downwards.

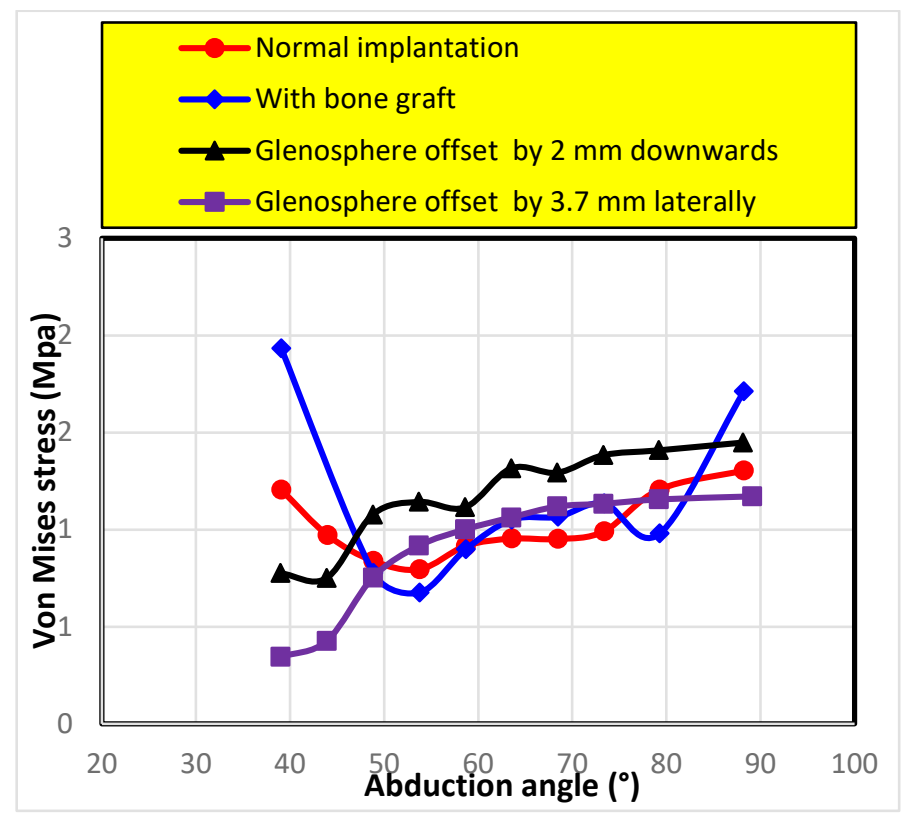

Fig. 9 Evolution of maximum stresses in the support with screws for different configurations according to the abduction angle 
Figure 10 illustrates the distribution of von Mises stresses in the support at the final position of the abduction movement for the four configurations. It can be seen that for the configurations (a), (b) and (c), the maximum stress is located at the outer peripheral contour of the support, while for the configuration (d), it is located near the central hole of the support. This can be attributed to the effect of the abduction movement which differs from one configuration to another.

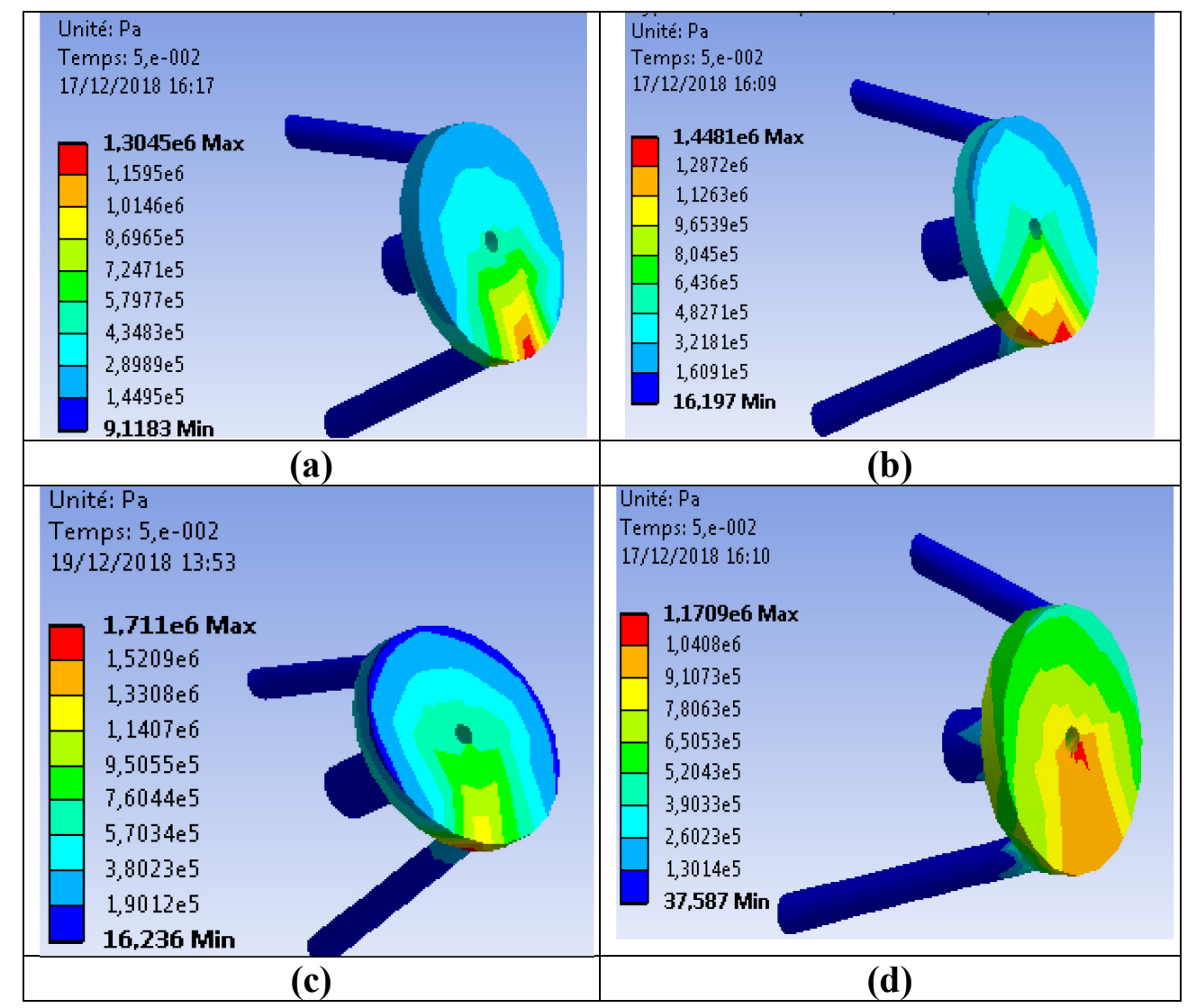

Fig. 10 Distribution of von Mises stresses in the support with screws for different configurations at the end of abduction motion: (a) a normal implantation with a medialized center of rotation, (b) with glenosphere offset from the center of rotation by $2 \mathrm{~mm}$ downwards, (c) with glenosphere lateralized by $10 \mathrm{~mm}$ (BIO-RSA) and (d) with glenosphere offset by $3.7 \mathrm{~mm}$ laterally

\section{Discussion}

Improvements to the Grammont prosthesis are intended to avoid the disadvantages and complications of the latter, in particular the notch problem. These modifications consist in lowering and/or lateralization of the prosthesis, either through the use of technical devices (lowering of the metaglene according to Nyffeler [25] or bone lateralization according to Boileau [26]), or by modifying the design of the implants by seeking the biomechanical compromise (lateralized rotation center, lowering, eccentric) [27].

Clinically, the literature is controversial with regard to the Range Of Motion (ROM). A recent study by Collin et al. [27] reports that bending forward is more important after BIO-RSA than after a neutral glenosphere of $36 \mathrm{~mm}$ [24]. As well as the rate of notch is $75 \%$ demineralized for reverse shoulder prostheses with a medialized center of rotation of $45 \%$ for BIO-RSA prostheses [28].

According to the work of Boileau et al. [29], prostheses with a lateralized glenosphere (the configuration (d) in Figure 5) have the disadvantage of increasing the torque or shear force applied to the glenoid component, which accelerates the wear of the glenosphere and the polyethylene cup.

Numerically, Denard et al. [30] have shown the interest of simulations to improve implantation methods. In our study we wanted to go further by getting as close as possible to the biomechanics of movement and taking care to validate the numerical model. The validation of the model was 
performed using results from the literature, which allowed us to give credibility to our simulations and results of stresses and contact forces.

Our study confirms the hypothesis that the glenoid configuration has an effect on the biomechanical behavior of the reverse shoulder prosthesis. In particular, the evolution of the forces and the maximum stresses observed on the glenosphere as a function of the abduction angle of the arm.

Figure 11 shows the variation of the maximum forces recorded at the level of the glenoid for the four configurations. It is clear that the forces are higher for the prosthesis with a glenoid offset by 3.7 $\mathrm{mm}$ laterally (configuration (d)), and are minimal for the prosthesis with a medialized center of rotation glenoid (configuration (a)). In addition, it can be observed that the prosthesis which has a behavior closer to that of the prosthesis with a medialized rotation center glenoid is the prosthesis with bone graft (BIO- RSA) (configuration (b)).

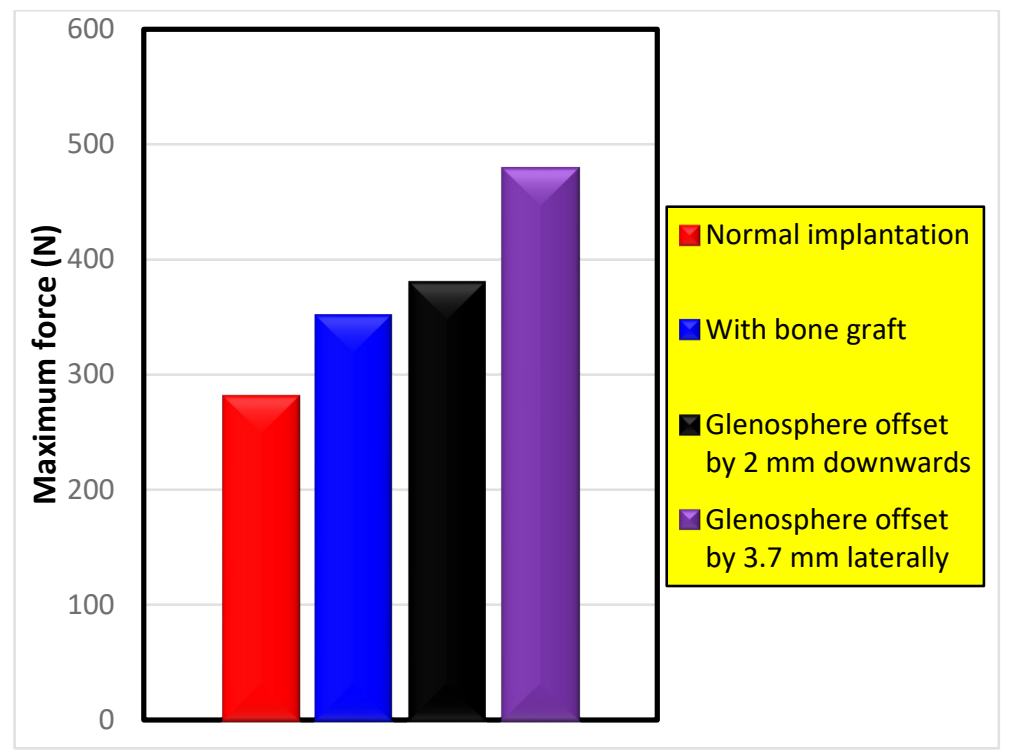

Fig. 11 Maximum values of the forces recorded on the glenoid.

The results presented in Figure 12 show that the maximum stresses at the glenosphere level are very high in the case of configuration (d) compared to the prosthesis with a medialized center of rotation glenoid (configuration (a)).

For the support with screws, figure 13 shows that the maximum value is obtained in the case of a glenosphere with bone graft, while a slight difference was noted for the other configurations. It is also important to notice that the stresses values obtained for the four configurations are permissible.

Finally, depending on the choice of the different indicators, this or that method appears more or less as the best implantation. For example, the BIO-RSA method minimizes contact forces on the glenoid, while the implantation with a glenosphere offset from the center of rotation bay $2 \mathrm{~mm}$ downwords (configuration (b)) minimizes stresses. However, on average on the different indicators, method (b) is the closest to the normal method. 


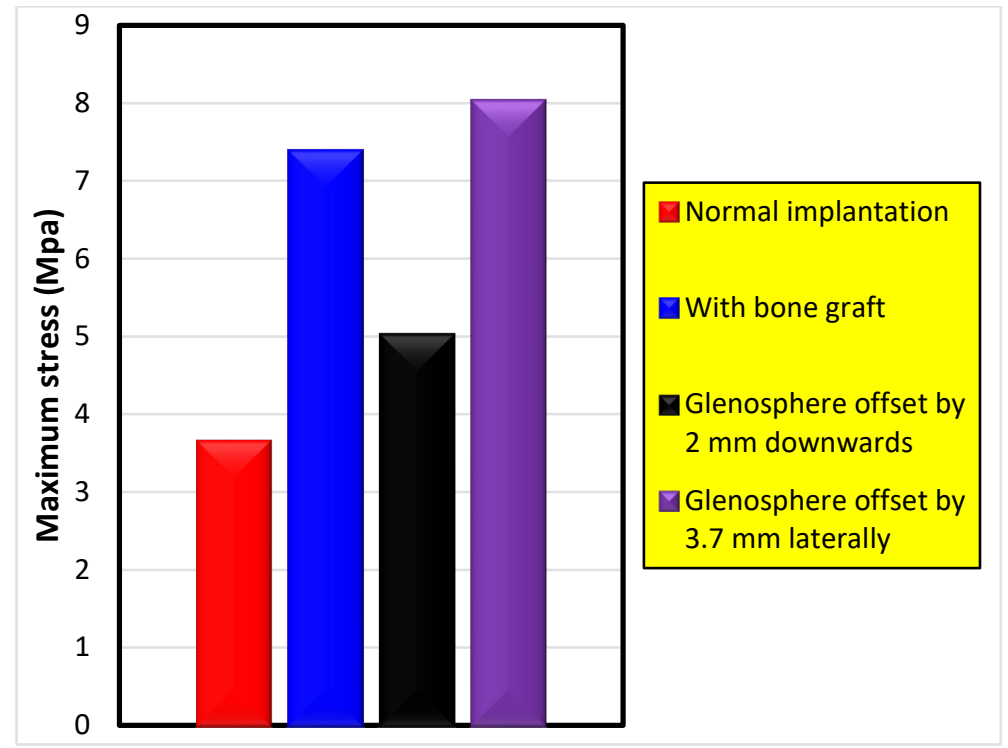

Fig. 12 Illustration of the maximum stresses on the glenosphere during abduction movement

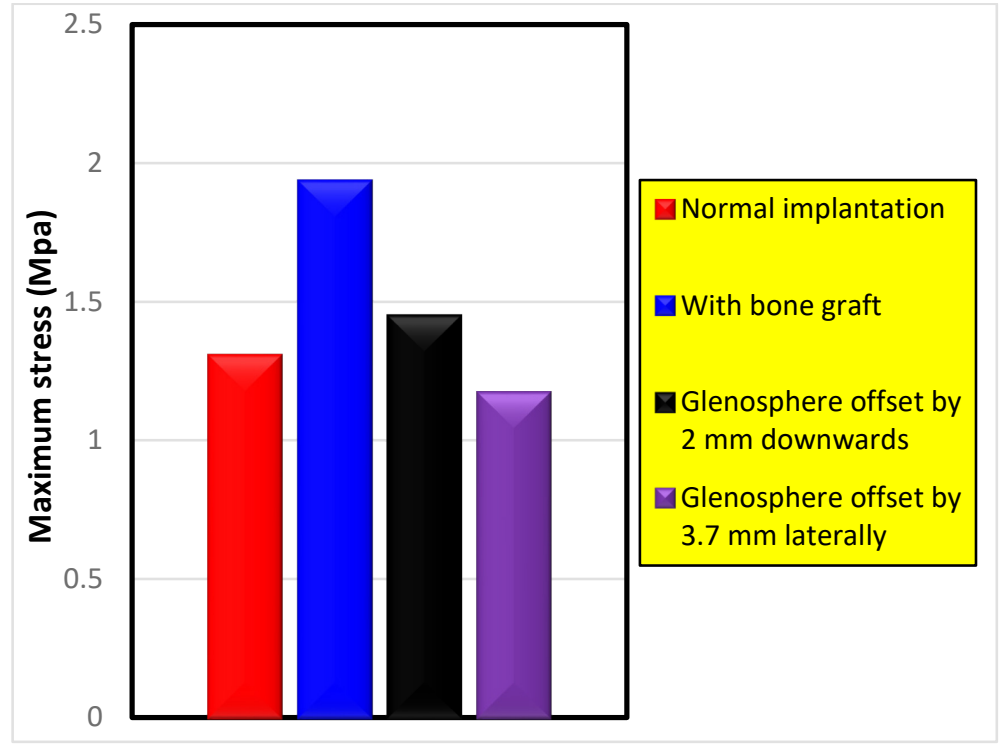

Fig. 13 Illustration of the maximum stresses in the supports with screws

\section{Conclusion}

In this work, the biomechanical behaviors of four different RSA configurations were studied in order to find an adequate solution to the notch problem and highlight their influences on the prosthesis performance. The numerical models were developed through three main phases: (i) 3D reconstruction of the bones of the intact shoulder, (ii) design of the different components of the reverse prosthesis, (iii) implantation of the prosthesis according to different configurations and finite element analysis.

After the validation of the numerical model in the case of normal implantation with a medialization center of rotation using results from the literature, a numerical investigation of the biomechanical behaviors of three new implantation methods under the action of the arm abduction movement is presented. The effect of these implantation methods on the performance of the reverse shoulder prosthesis have been highlighted.

From the obtained results, we can draw the following conclusions:

- The contact force at the glenoid surface increases with the increase of the abduction angle.

- The configuration that appears closest to the results obtained by the normal implantation, in terms of contact forces is that bone graft ( configuration (b)), however, in terms stresses is that with glenosphere offset from the center of rotation by $2 \mathrm{~mm}$ downward. 
- Minimum contact force values during arm abduction movement are obtained in the case of normal implantation (with a medializaed center of rotation).

In addition, it can be noted that this study has highlighted the influence of the implantation method on the performance of the reverse shoulder prosthesis and consequently, it presents a very useful tool to assist in the suitable choice of the implantation method for the latter.

\section{References}

[1] J. Wang, Y. Zhu, F. Zhang, W. Chen, Y. Tian, Y. Zhang, Meta-analysis suggests that reverse shoulder arthroplasty in proximal humerus fractures is a better option than hemiarthroplasty in the elderly. J Int Orthop. (2015).

[2] D. Bachman, J. Nyland, R. Krupp; Reverse-total shoulder arthroplasty cost-effectiveness: a quality-adjusted life year's com-parison with total hip arthroplasty. World J Orthop. 7(2): (2016) $123-127$

[3] M. Shafi, JJ. Jeong, JY. Ha; Reverse total shoulder arthroplasty in the treatment of chronic anterior fracture dislocation complicated by a chronic full thickness retracted rotator cuff tear in an elderly patient. J Orthop Science. 21(2) (2016) $237-240$.

[4] W. Liou, Y. Yang, G. R. Petersen-Fitts, , D. J. Lombardo, S. Stine, V. J. Sabesan,. Effect of lateralized design on muscle and joint reaction forces for reverse shoulder arthroplasty. $\mathrm{J}$ Shoulder Elbow Surg. (2017) 564-572.

[5] J. Kurowicki, J. J.Triplet, E. Momoh, M. A.Moor, J. C.Levy; Reverse shoulder prosthesis in the treatment of locked anterior shoulders: a comparison with classic reverse shoulder indications; J Shoulder Elbow Surg. (2016) 1954-1960.

[6] Guery J, Favard L, Sirveaux F, Oudet D, Mole D, Walch G. Reverse total shoulder arthroplasty. Survivorship analysis of eighty replacements followed for five to ten years. J Bone Joint Surg Am. (2006) 1742-1747.

[7] JS. Day, E. Lau, KL. Ong, GR. Williams, ML. Ramsey, SM. Kurtz. Prevalence and projections of total shoulder and elbow arthroplasty in the United States to 2015. J Shoulder Elbow Surg. (2010) 5-20.

[8] RW. Westermann, AJ. Pugely, CT. Martin, BR. Gao Y,Wolf, CM. Hettrich. Reverse shoulder arthroplasty in the United States: a comparison of national volume, patient demographics, complications, and surgical indications. J Iowa Orthop (2015) 1-7.

[9] JL. Berliner, A. Regalado-Magdos, BT. Ma CB, Feeley. Biomechanics of reverse total shoulder arthroplasty. J Shoulder Elbow Surg; (2015) 50-60.

[10] P. Boileau, JF. Gonzalez, C. Chuinard, R. Bicknell, G. Walch. Reverse total shoulder arthroplasty after failed rotator cuff surgery. J Shoulder Elbow Surg (2009) 600-606.

[11] F. Sirveaux, L. Favard, D. Oudet, D. Huquet, G. Walch, D. Molé. Grammont inverted total shoulder arthroplasty in the treatment of glenohumeral osteoarthritis with massive rupture of the cuff. Results of a multicenter study of 80 shoulders. J Bone Joint Surg Br (2004) 88-95.

[12] A. Kontaxis, GR. Johnson. The biomechanics of reverse anatomy shoulder replacement-a modelling study. Clin Biomech (2008) 54-60.

[13] A. Boulahia, TB. Edwards, G. RV walch, Baratta. Early results of reverse design prosthesis in the treatment of the shoulder in elderly with a large rotator cuff tear. Orthop (2002) 25-33.

[14] J. Guery, L. Favard, F. Sirveaux, D. Oudet, D. Mole, G. Walch. Reverse total shoulder arthroplasty. Survivorship analysis of eighty replacements followed for five to ten years. J Bone Joint Surg Am (2006) 1742-1747 
[15] M. Rittmeister, F. Kerschbaumer. Grammont reverse total shoulder arthroplasty in patients with rheumatoid arthritis and nonrecostructible rotator cuff lesions. J Shoulder Elbow Surg (2001) $17-22$.

[16] PM. Gramment, E. Baulot. Delta shoulder prosthesis for rotator cuff rupture. Orthopedics (1993) 65-68

[17] S. Guterrez, RM. Greiwe, MA. Frankle, S. Siegal, Lee WE III. Biomechanical comparison of component position and hard-ware failure in the reverse shoulder prosthesis. J J Shoulder Elbow Surg (2007) S9-S12.

[18] N. Kirzn, E. Paul, A. Moaveni, Reverse shoulder arthroplasty vs BIO-RSA: clinical and radiographic outcomes at short term follow-up, J Orthop Surg Res (2018) 18-23.

[19] S. Mebarki, B. Aour, F. Jourdan, E. Malachanne, A.H. Belaghit, A Study of the Biomechanical Behavior of the Implantation Method of Inverted Shoulder Prosthesis (BIO-RSA) under Different Abduction Movements. J BIOEBG (2019) 6-19.

[20] W. Pomwenger, K. Entacher, H. Resch, S.G. Peter, Multi patient finite element simulation ofkeeled versus pegged glenoid implant designs in shoulder arthroplasty, Med Biol Eng Comput (2015) 781-790.

[21] Astier, V., Thollon, L., Arnoux, P. J., Mouret, F. and Brunet, C., Development of a finite element model of the shoulder: application during a side impact', INT. J. CRASHWORTHINESS (2008) 301-312.

[22] A. Terrier, P. Büchler, A. Farron, Influence of glenohumeral conformity on glenoid stresses after total shoulder arthroplasty, J Shoulder Elbow Surg (2006) 515-520.

[23] N. Wuelker, C.J. Wirth, W. Plitz and B. Roetman, A dynamic shoulder model: reliability testing and muscle force study. J Biomech (1995) 489-499.

[24] Athwal GS, MacDermid JC, Reddy KM, Marsh JP, Faber KJ, Drosdowech D. Does bony increased-offset reverse shoulder arthroplasty decrease scapular notching? J Shoulder Elbow Surg (2015) 68-73.

[25] RW. Nyffeler, CML. Werner, C. Gerber, Biomechanical relevance of glenoid component positioning in the reverse Delta III total shoulder prosthesis, J Shoulder Elbow Surg (2004) 524528

[26] P.Boileau, F.Balg The reverse shoulder prosthesis: biomechanical principles, concept and evolution. (2008) 526-530.

[27] P. Collin, X. Liu, PJ. Denard, S. Gain, A. Nowak, A. Ladermann; Standard versus bony increased-offset reverse shoulder arthroplasty: a retrospective comparative cohort study. J Shoulder Elb Surg (2017) 59-64.

[28] A. Lädermann, Patrick J. Denard, Pascal Boileau, Alain Farron, Pierric Deransart, Gilles Walch. What is the best glenoid configuration in onlay reverse shoulder arthroplasty? (2018) 13391346.

[29] P. Boileau, G. Moineau, Y. Roussanne, K. O’Shea. Bony increased-offset reversed shoulder arthroplasty: minimizing scapular impingement while maximizing glenoid fixation. Clin Orthop Relat Res (2011) 11-17.

[30] Denard et al, Finite Element Analysis of Glenoid-Sided Lateralization in Reverse Shoulder Arthroplasty, J Orthop Res (2017) 1548-1555. 